\title{
Design and Simulation of Copper-based Multimetal-contact RF MEMS Switch
}

\author{
Zhaoqun Jiang $^{\mathrm{a}}$, Zhuhao $^{\mathrm{b}}$ Gong, Zewen Liu ${ }^{\mathrm{c}}$ \\ Institute of Microelectronics, Tsinghua University, Beijing, China \\ ajzq13@mails.tsinghua.edu.cn, ${ }^{\mathrm{b}}$ gongzh12@mails.tsinghua.edu.cn, ${ }^{\mathrm{c}}$ liuzw@tsinghua.edu.cn
}

Keywords-RF MEMS switch, material hardness, multilayer stack, copper

\begin{abstract}
This paper presents the design and simulation of metal-contact RF micro electromechanical system (MEMS) switch based on the multilayer metal stack $\mathrm{Au} / \mathrm{Cu} / \mathrm{Au}$. The novel concept of $\mathrm{Au} / \mathrm{Cu} / \mathrm{Au}$ contact metallization is introduced to increase the hardness of the contact material. The mechanical and RF characteristics of the designed switch are investigated by Coventor Ware and ANSOFT HFSS. The simulation results indicate that the pull-in voltage is $32 \mathrm{~V}$, the closing time is $25 \mu \mathrm{s}$, and the opening time is $18 \mu \mathrm{s}$. The insertion loss is better than $-0.12 \mathrm{~dB}$ and isolation is better than $-25 \mathrm{~dB}$ up to $20 \mathrm{GHz}$.
\end{abstract}

\section{Introduction}

Radio frequency micromechanical system (RF MEMS) switches are promising substitutes for the conventional ones, because they offer numerous advantages over their mechanical and solid-state counterparts, such as low insertion loss, high linearity, high isolation and low power consumption [1].Metal-contact MEMS switches maintain excellent performance over a very large bandwidth from dc to tens of gigahertz. Despite these evident advantages of Metal-contact MEMS switches, some reliability issues prohibit their large-scale application.

Contact stiction and contact degradation are the main reliability issues [2], which are related to the metal-to-metal contact area. The low hardness of gold, widely used as contact material due to its low resistivity and chemical inertness, results in material transfer and wear, and ultimately causes switch failure. A common strategy to overcome this problem is to use harder metal and its alloy as contact material. Many research groups have paid a considerable effort to the material selection for MEMS switch, but none of them have been proven to be totally successful. Many harder metal such as $\mathrm{Ru}, \mathrm{Rl}$, Ir, and their alloys tend to react when exposed to ambient atmosphere and form a nonconductive contamination film during cycling, which makes the contact resistance increase rapidly. The lifetime of these switches utilizing harder metal as contact material is much shorter than Au-Au contact switch [3,4].

A novel multilayer stack $\mathrm{Au} / \mathrm{Cu} / \mathrm{Au}$ is introduced to increase the hardness of contact material. Compared to $\mathrm{Au}, \mathrm{Cu}$ is a much harder material and higher hardness can reduce contact wear to offer the advantage of better reliability. In addition to higher hardness, $\mathrm{Cu}$ offers the higher electrical and thermal conductivity as compared to $\mathrm{Au}$. In order to protect $\mathrm{Cu}$ from oxidation during the fabrication process, thin film of $\mathrm{Au}$ is introduced to coat the surfaces of $\mathrm{Cu}$.

\section{Mechanical design and simulation}

Cantilever beam based on multilayer stack is presented in Fig.1. Deriving the spring constant of cantilever beam is the foundation to understand the mechanical properties of RF MEMS switch. The spring constant of the cantilever beam with concentrated vertical force 


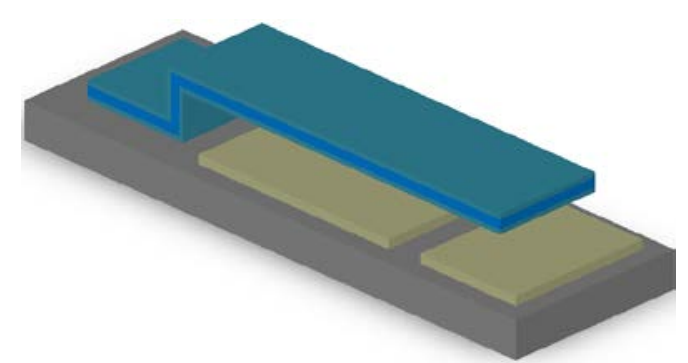

at the tip of the beam is given as

Fig.1 Cantilever beam composed of different layers

$$
k_{e}=\frac{E_{e} w}{4}\left(\frac{t}{l}\right)^{3}
$$

where $t$ is the thickness, $l$ is the length and $w$ is the width of the beam. $E_{e}$ is the equivalent Young's modulus of beam composed of different layers, each with $E_{n}$ and $t_{n}$, and can be expressed as

$$
E_{e}=\frac{\sum E_{n} t_{n}}{t}
$$

Substituting (2) into (1) gives

$$
k_{e}=\frac{E_{A u} t_{A u}+E_{C u} t_{C u}}{t} \frac{w}{4}\left(\frac{t}{l}\right)^{3}
$$

In order to investigate the effect of the thickness of different layers on the equivalent spring constant $k_{e}, k_{e}$ is normalized by $k_{C u}$

$$
k_{e}^{\prime}=\frac{79\left(t_{A u} / t_{C u}\right)+128}{128\left(t_{A u} / t_{C u}+1\right)}
$$

In equation (4), the modulus of $\mathrm{Au}$ and $\mathrm{Cu}$ are set as 79GPa and 128GPa. Fig.2 shows the variation of $\mathrm{k}_{e}^{\prime}$ with $t_{A u} / t_{C u}$ from 0 to 1 . In fact, $\mathrm{Cu}$ is the major part of the volume of multilayer stack, i.e., $t_{A u} / t_{C u}<1$, which means that the thickness of Au-film has little effect on the spring constant of multilayer-stack cantilever beam, so we use (1) instead of (3) to design the cantilever beam.

The pull-in voltage of the cantilever RF MEMS switch is given in [1]

$$
V_{p}=\sqrt{\frac{8}{27} \frac{k_{e} g_{0}^{3}}{\varepsilon_{0} A}}
$$

where $g_{0}$ is the gap between the beam and actuation electrode, $A$ is the area of the upper electrode.

The designed switch is presented in Fig 3, whose parameters are listed in Table 1. The mechanical properties of the designed switch is simulated by CoventorWare. The pull-in voltage is $32 \mathrm{~V}$ and the switching time simulation result is presented in Fig.4. The closing time is $25 \mu$ s, and the opening time is $18 \mu \mathrm{s}$, which are fast enough for the automated reconfiguration of telecommunication networks [5].

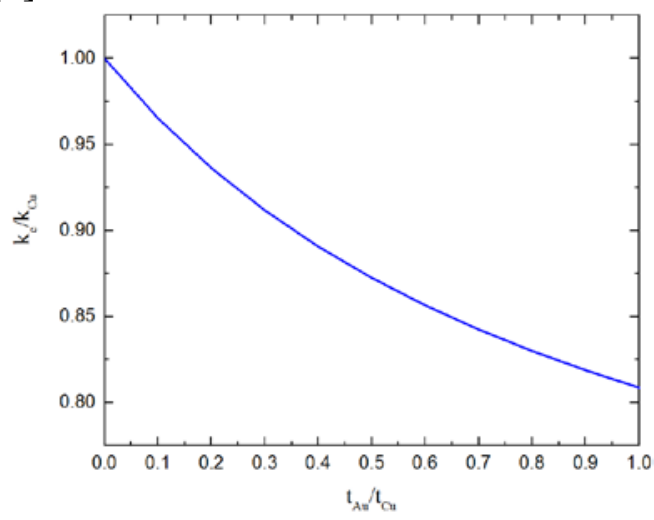

Fig.2 Normalized spring constant $\mathrm{k}_{e}^{\prime}$ versus $t_{A u} / t_{C u}$ 


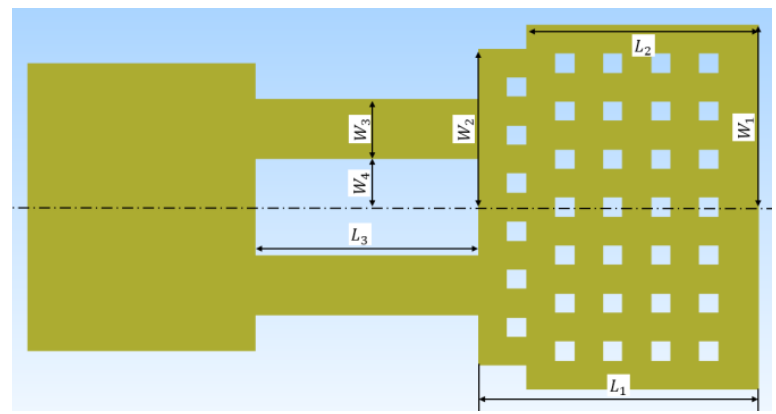

Fig.3 Schematic of the designed switch

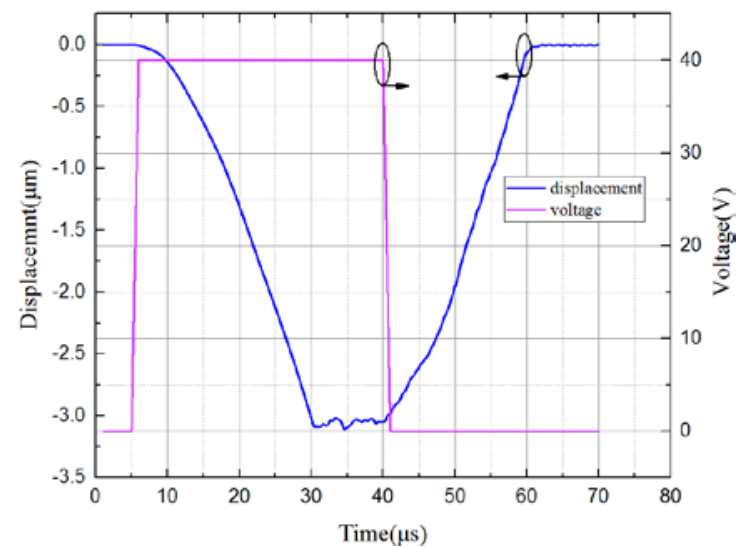

Fig.4 Switching time simulation of the designed switch

Table 1 Parameters of the switch dimension

\begin{tabular}{cc}
\hline \hline Parameters & Value $(\mu \mathrm{m})$ \\
\hline$t$ & 2.4 \\
$H$ & 700 \\
$g_{0}$ & 3 \\
$L_{1}$ & 117 \\
$L_{2}$ & 97 \\
$L_{3}$ & 93 \\
$W_{1}$ & 76 \\
$W_{2}$ & 66 \\
$W_{3}$ & 25 \\
$W_{4}$ & 20 \\
\hline \hline
\end{tabular}

\section{RF design and simulation}

For the further connection with other RF components, coplanar waveguide (CPW) is designed as the feed configuration and the 700- $\mu$ m-thick SCHOTT BOROFLOAT ${ }^{\circledR} 33$ (B33) glass is utilized as substrate with dielectric constant of 4.6. The dimensions of CPW, $120-\mu \mathrm{m}$ signal-line width and $19-\mu \mathrm{m}$ gap to ground lines, result in a characteristic impedance of $49.38 \Omega$ with a conductor thickness of $2.4 \mu \mathrm{m}$.

The schematic of the designed switch with CPW is shown in Fig.5.

The most important RF characteristics of switch are isolation in upstate and insertion loss in downstate. Isolation is determined by the overlapping area and the suspension height of the upper electrode, which are $19 \mu \mathrm{m} \times 20 \mu \mathrm{m}$ and $3 \mu \mathrm{m}$ respectively in the designed switch.

Insertion loss is determined by the contact resistance, which arises from the rough property of the contact surfaces, because only the higher asperities distributed in the surfaces enter into contact when the contact makes. Typically, the calculation of the contact resistance between two rough surfaces is very difficult because multiple asperities are involved in the contact making. These asperities have different contact interference and may interact with each other. In order to calculate the contact resistance, we use the asperity contact model to represent the rough surface contact.

For the $\mathrm{Au} / \mathrm{Cu} / \mathrm{Au}$ multilayer stack, the total contact resistance consists of two parts, the 
constriction resistance term $R_{c}$ and film

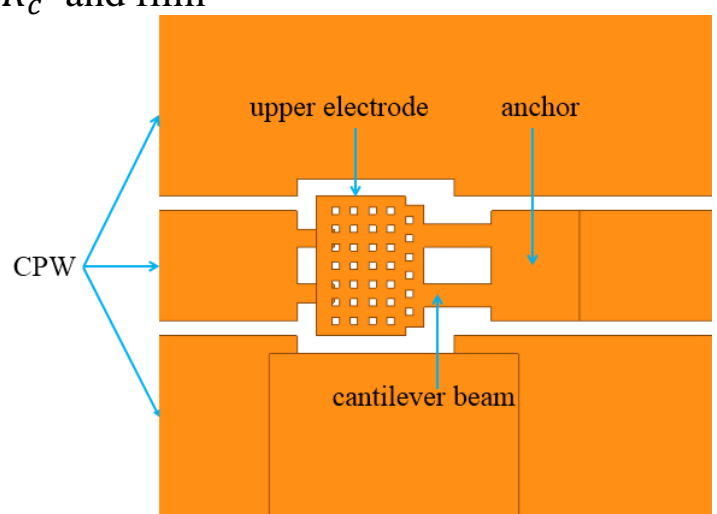

Fig.5 The designed switch with CPW configuration

resistance $R_{f}$

$$
R=R_{c}+R_{f}=\frac{\rho}{2 a}+\frac{\rho_{f} d}{\pi a^{2}}
$$

with $a$ being the radius of the contact spot; $\rho$ being the resistivity of the major part of the asperity, here $\mathrm{Cu} ; \rho_{f}$ being the resistivity of film, here Au; $d$ being the thickness of the film. When the mean free path $l_{e}$ is comparable to radius of the contact spot, the constriction resistance consists of two components, Maxwell spreading resistance $R_{M}$ due to the lattice scattering and Sharvin resistance $R_{S}$ due to boundary scattering. The constriction resistance in small contact spot is given in [6]

$$
R_{c}=\frac{1+0.83\left(l_{e} / a\right)}{1+1.33\left(l_{e} / a\right)} \frac{\rho}{2 a}+\frac{4 \rho l_{e}}{3 \pi a^{2}}
$$

Substituting (7) into (6) results in

$$
R=\frac{1+0.83\left(l_{e} / a\right)}{1+1.33\left(l_{e} / a\right)} \frac{\rho}{2 a}+\frac{4 \rho l_{e}}{3 \pi a^{2}}+\frac{\rho_{f} d}{\pi a^{2}}
$$

We assume that the deformation of the asperity is fully plastic, so the relationship between contact area $\pi a^{2}$ and contact force is given in [7]

$$
F=2 H A_{C}=2 H \pi a^{2}
$$

which results in

$$
a=\sqrt{\frac{F}{2 H \pi}}
$$

According to (8) and (9), we can derive the relationship between the contact force and contact resistance with different coating thickness, which is plotted in Fig.6. The hardness used for the calculation are obtained by nanoindentation test. The test equipment is Nanoindenter XP (MTS System Corporation) with a Berkovich tip and the working mode is dynamic contact module(DCM). The test results are $H_{A u}=0.23 G P a, H_{C u}=1.69 G P a$. The mean free path of $\mathrm{Cu}$ is set as $51 \mathrm{~nm}$. As demonstrated in Fig.6, the contact resistance is about $0.2 \Omega$ if the contact force is larger than $50 \mu N$. 


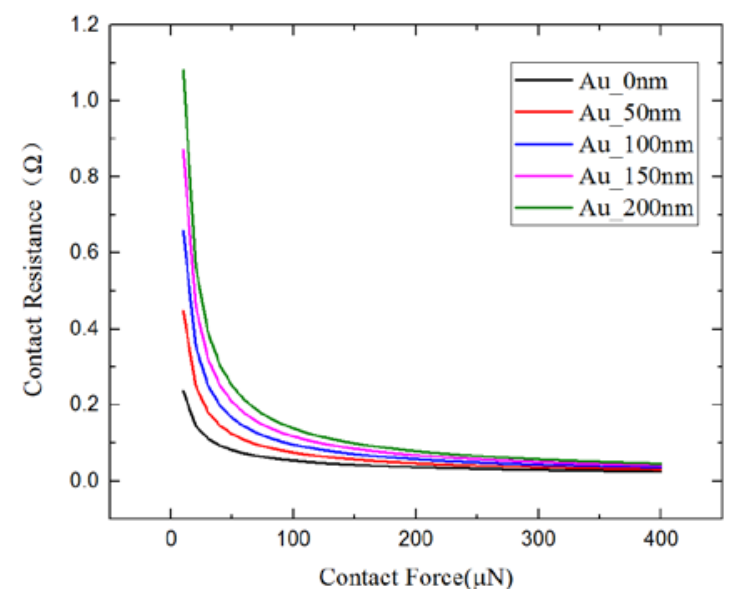

Fig.6 Contact resistance versus contact force

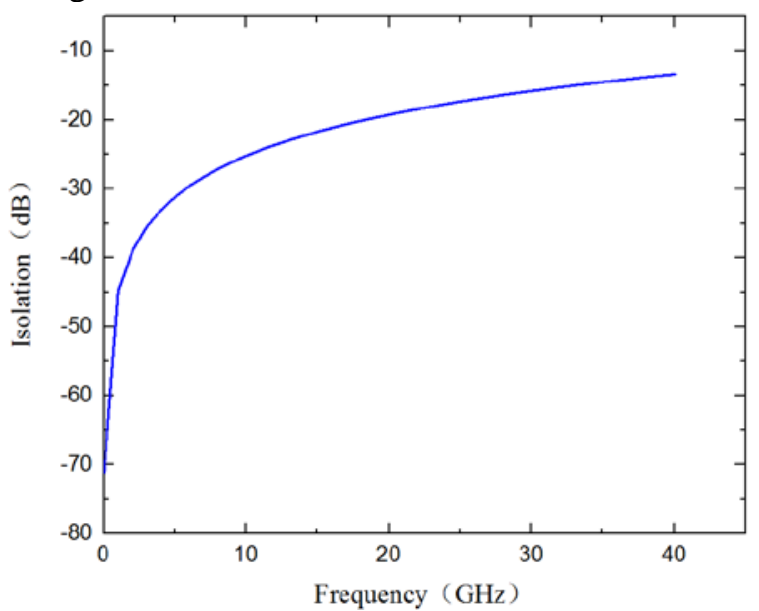

(a) isolation

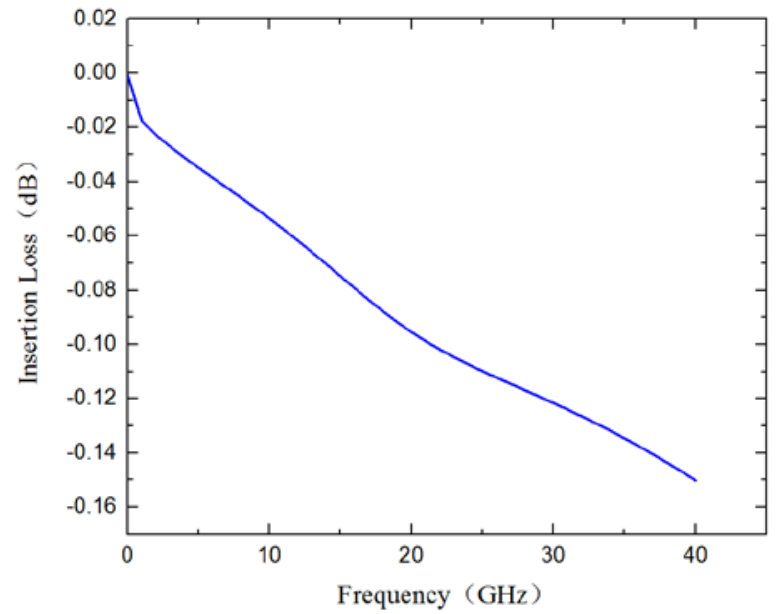

(b) insertion loss

Fig.7 RF characteristics simulation results of the designed switch

Based on the analysis above, the contact resistance is set as $0.2 \Omega$ when simulating the insertion loss. The RF characteristics of the designed switch are investigated by ANSOFT HFSS. The simulation results are presented in Fig.7. Simulation results indicate that isolation is better than $-25 \mathrm{~dB}$ and insertion loss is better than $-0.12 \mathrm{~dB}$ up to $20 \mathrm{GHz}$.

\section{Conclusion}

This paper introduces a novel multilayer metal stack $\mathrm{Au} / \mathrm{Cu} / \mathrm{Au}$ to design $\mathrm{RF}$ MEMS switch and details the design and simulation of the switch based on the multilayer stack. The pull-in voltage is $32 \mathrm{~V}$, the closing time is $25 \mu \mathrm{s}$, the opening time is $18 \mu \mathrm{s}$. The isolation is better than $-25 \mathrm{~dB}$ and the insertion loss is better than $-0.12 \mathrm{~dB}$ up to $20 \mathrm{GHz}$. The simulation results indicate that the designed 
switch has excellent switching performance.

\section{Reference}

[1] G. M. Rebeiz, RF MEMS: Theory, Design, and Technology, second ed., Wiley, Hoboken, New Jersey 2002.

[2] B.F. Toler, R.A. Coutu Jr, and J.W. McBride, A review of micro-contact physics for microelectromechanical systems (MEMS) metal contact switches. Journal of Micromechanics and Microengineering, 2013, 23(10):103001.

[3] D. A. Czaplewski, C.D. Nordquist, and C.W. Dyck, Lifetime limitations of ohmic, contacting RF MEMS switches with Au, Pt and Ir contact materials due to accumulation of 'friction polymer' on the contacts, Journal of Micromechanics and Microengineering, 2012, 22(10):105005

[4] L. Chen, Z. J. Guo and N. E. McGruer, Contact resistance study of noble metals and alloy films using a scanning probe microscope test station, Journal of Applied Physics, 2007,102(7): 074910

[5] J Oberhammer, G Stemme, Single-chip MEMS 5x5 and 20x20 double-pole single-throw switch arrays for automating telecommunication networks, Journal of Micromechanics and Microengineering, 2008, 18(1): 015014

[6] B. Nikolic and P.B. Allen, Electron transport through a circular constriction, Phys. Rev. B, Condens. Matter, 1999, 60(6): 3963-3969.

[7] W.R. Chang, An elastic-plastic contact model for a rough surface with an ion-plated soft metallic coating[J]. Wear, 1997, 212(2):229-237. 